Boise State University

ScholarWorks

Kinesiology Faculty Publications and Presentations

Department of Kinesiology

5-1-2011

\title{
The Effects of Repetitive Drop Jumps on Impact Phase Joint Kinematics and Kinetics
}

Joshua T. Weinhandl

University of Wisconsin-Milwaukee

Jeremy D. Smith

University of Northern Colorado

Eric L. Dugan

Boise State University 


\title{
The Effects of Repetitive Drop Jumps on Impact Phase Joint Kinematics and Kinetics
}

\author{
Joshua T. Weinhandl, Jeremy D. Smith, and Eric L. Dugan
}

\begin{abstract}
The purpose of the study was to investigate the effects of fatigue on lower extremity joint kinematics, and kinetics during repetitive drop jumps. Twelve recreationally active males $(n=6)$ and females $(n=6)$ (nine used for analysis) performed repetitive drop jumps until they could no longer reach $80 \%$ of their initial drop jump height. Kinematic and kinetic variables were assessed during the impact phase $(100 \mathrm{~ms})$ of all jumps. Fatigued landings were performed with increased knee extension, and ankle plantar flexion at initial contact, as well as increased ankle range of motion during the impact phase. Fatigue also resulted in increased peak ankle power absorption and increased energy absorption at the ankle. This was accompanied by an approximately equal reduction in energy absorption at the knee. While the knee extensors were the muscle group primarily responsible for absorbing the impact, individuals compensated for increased knee extension when fatigued by an increased use of the ankle plantar flexors to help absorb the forces during impact. Thus, as fatigue set in and individuals landed with more extended lower extremities, they adopted a landing strategy that shifted a greater burden to the ankle for absorbing the kinetic energy of the impact.
\end{abstract}

Keywords: fatigue, landing, power, energy absorption, biomechanics

Landing tasks place high mechanical demands on the joints and tissues of the lower extremity. During vertical landings, the lower extremity extensors produce torque and do work to decrease the body's vertical momentum (Schot \& Dufek, 1993). This can be accomplished in several different ways and the lower body joint configuration at initial contact has a major influence on energy absorption during landing tasks (DeVita \& Skelly, 1992; Kulas et al., 2006; Zhang et al., 2000). Soft landings are generally characterized by greater amounts of flexion and energy absorbed by the knee and hip extensors, while stiff landings exhibit high ankle and knee energy absorption with reductions in hip energy absorption (Decker et al., 2003; DeVita \& Skelly, 1992; Zhang et al., 2000) and in the total summated work of the lower extremity joints (DeVita \& Skelly, 1992). However, while changes in kinematics and kinetics in response to changes in landing technique are well documented, the effects of neuromuscular fatigue on lower extremity biomechanics are not clearly understood.

Neuromuscular fatigue, which can be defined as a decreased capacity of muscle fibers to absorb energy and produce force, might be a contributing factor in musculoskeletal injuries during prolonged activity.

Joshua T. Weinhandl (Corresponding Author) is with the Department of Human Movement Sciences, University of Wisconsin-Milwaukee, Milwaukee, WI. Jeremy D. Smith is with the School of Sport and Exercise Science, University of Northern Colorado, Greeley, CO. Eric L. Dugan is with the Department of Kinesiology, Boise State University, Boise, ID.
There is evidence that neuromuscular fatigue leads to changes in landing postures and subsequent compensatory landing strategies (Chappell et al., 2005; Coventry et al., 2006; Fagenbaum \& Darling, 2003; Kernozek et al., 2008; Madigan \& Pidcoe, 2003; McLean et al., 2007; Pappas et al., 2007). Decker et al. (2003) theorized that in the presence of muscular fatigue, a more erect landing may increase injury risk. The reduction of force producing capabilities of the muscles coupled with a more erect landing strategy may produce a mechanical disadvantage for the hamstring muscles and allow the quadriceps muscles to pull the tibia anteriorly resulting in larger anterior cruciate ligament (ACL) forces (Pandy $\&$ Shelburne, 1997). It is plausible that increased plantar flexion during a fatigued state may serve as a protective mechanism for the passive structures of the knee joint, allowing for greater energy absorption through the ankle plantar flexors and less reliance on the knee extensor musculature to dissipate energy during the landing (Self \& Paine, 2001). Conversely, landing with greater knee flexion and less ankle plantar flexion at ground contact may indicate that the knee is more prepared to transfer energy up the kinetic chain to the larger and more proximal muscles such as the hip extensors (Self \& Paine, 2001). Thus, preplanned muscular landing strategies and the position of the lower extremity joints at ground contact may collectively influence the magnitude of the peak vertical ground reaction force (VGRF) (Schot \& Dufek, 1993). Therefore, the reduced capacity of the musculature to produce force and thus attenuate the peak VGRF during landing may place individuals at a higher risk of injury. 
The research on landing mechanics and associated injury risks is vast; however, the results concerning changes in landing strategies as a result of fatigue have been equivocal. Epidemiological studies support the notion that fatigue - along with playing intensity, aggression and impairment in cognitive function-may be predisposing factors responsible for the increased number of injuries at the end of games or halves (Hawkins et al., 2001; Rahnama et al., 2002). It appears that fatigue leads to changes in lower extremity kinematics during landing (Chappell et al., 2005; Coventry et al., 2006; Fagenbaum \& Darling, 2003; Kernozek et al., 2008; Madigan \& Pidcoe, 2003; McLean et al., 2007; Pappas et al., 2007), accompanied by a reduced capacity of the musculature to dissipate energy (Decker et al., 2003; Self \& Paine, 2001) which potentially exposes the passive structures of the joints to greater stresses as they must absorb more energy (DeVita \& Skelly, 1992). Despite this, a very limited number of studies have examined the effect of fatigue on biomechanical variables during drop jumps (McLean et al., 2007; Moran \& Marshall, 2006) and none have used a fatigue protocol consisting of repetitive drop jumps. Therefore, the purpose of this study was to investigate the effects of fatigue on lower extremity joint kinematics, and kinetics during repetitive drop jumps.

\section{Methods}

\section{Subject Population}

Twelve healthy, recreationally active university students, male $(n=6,22 \pm 2$ years, $89.2 \pm 7.1 \mathrm{~kg}, 1.81 \pm 0.13 \mathrm{~m})$ and female $(n=6,22 \pm 1$ years, $59.9 \pm 8.3 \mathrm{~kg}, 1.66 \pm 0.06$ $\mathrm{m})$ volunteered to participate in this study. All subjects were currently involved in competitive intramural and/ or recreational sports sponsored by the university. Each subject participated in these activities at least three times per week and had not participated in varsity competition within the previous 18 months. All subjects also reported no history of lower extremity injury within the previous 18 months. Informed written consent was obtained from each subject before participation, and approval for the protocol was obtained from the university's Institutional Review Board.

\section{Experimental Protocol}

Subjects were asked to completed a warm-up protocol consisting of five minutes of treadmill walking at a selfselected pace, followed by an active warm-up of ten drop jumps from a height of $20 \mathrm{~cm}$. A successful drop jump required subjects to initially step off the raised platform and land with each foot making complete contact with a separate force plate. Immediately after landing, subjects were required to jump vertically, with the intent of achieving maximum height as quickly as possible. Next, motion data were measured during three maximal effort countermovement jumps and used to estimate jump height based on vertical displacement of the whole body center of mass (COM). The highest jump height was used to set the platform height during the experimental protocol.

Fatigue was induced by requesting the subjects to perform successive drop jumps from the raised platform every $20 \mathrm{~s}$. The use of arms during the drop jumps was eliminated by having the subjects hold a hollow PVC pipe across their shoulders. Apart from the arms, the drop jumps were performed according to the subjects' preferred style (i.e., to bend their knees to a freely chosen angle). The exercise was stopped when the subject could no longer reach $80 \%$ of the mean of their first five drop jumps for three consecutive trials. Drop jump height (DJH) was defined as the mean vertical displacement of the COM, and was monitored by a second researcher during the fatigue protocol on a second computer. Pilot data collections suggested that if a subject successfully completed 200 drop jumps without demonstrating a $20 \%$ decrement in DJH they likely did not give full effort during the protocol. Therefore, it was determined, a priori, that their data would be excluded from further data analysis. However, there is a small possibility that they were extremely fatigue resistant. In either case their data were not representative of those who exhibited a fatigue-induced change in performance which was the focus of the current study.

\section{Data Acquisition and Analysis}

Ground reaction force data were measure for each leg independently $(2400 \mathrm{~Hz})$ (AMTI, Watertown, MA). Three-dimensional coordinates of 39 markers were captured $(240 \mathrm{~Hz})$ with a 10-camera motion analysis system (Vicon, Lake Forest, CA). Marker coordinate data and ground reaction force data were filtered by fitting Woltring's quintic spline (Woltring, 1986) with a mean squared error setting of 15 to the data before running the biomechanical model (Vicon Plug-in-Gait, Oxford Metrics). Vicon's Plug-in-Gait model, used to derive kinematic and kinetic data of the lower extremity, is based on the methods described by Kadaba et al. (1990) and Davis et al. (1991) to define Cardan angles and construct a system of embedded coordinates from the marker set. Zero degrees at the three joints corresponded to an erect, standing position with the trunk, thigh, and leg in a straight line, and the foot at a right angle to the leg. Positive values were assigned for flexion at the hip and knee and dorsiflexion at the ankle. Internal joint moments were calculated for each joint by combining the kinematic and ground reaction force data with anthropometric data (Dempster, 1955) in an inverse dynamics solution. Positive moments were considered extensor at the hip and knee and plantar flexor at the ankle. Hip, knee and ankle muscle powers were also calculated as the product of the instantaneous joint moment and joint angular velocity. Positive muscle power indicated concentric action of the muscles and that energy was generated by the muscles. Negative muscle power indicated eccentric 
actions of the muscles and that energy was absorbed by the muscles.

Custom software, written in Matlab (The Mathworks Inc., Natick, MA), was used to calculate values for the dependent variables from the kinematic and kinetic profiles. Only kinematics and kinetics of the dominant leg, determined by asking subjects which leg they would use to kick a ball (right leg = 9; left leg = 3), were used in the analyses. Mechanical joint work, defined as the integral of the respective muscle power curve, was calculated for the hip, knee, and ankle during the impact phase of the landing. The impact phase consisted of the first $100 \mathrm{~ms}$ after initial contact with the ground (DeVita \& Skelly, 1992; Schot et al., 1994), which was defined as the instant VGRF exceeded a threshold of $20 \mathrm{~N}$ (Coventry et al., 2006). All kinetic parameters were scaled to body mass as in previous studies (Coventry et al., 2006; DeVita \& Skelly, 1992; Zhang et al., 2000). To accommodate for intersubject variations in the number of jumps completed, which ranged from 118 to 191 jumps, all dependent variables were normalized to 100 trials (Madigan \& Pidcoe, 2003). This normalization procedure, commonly used to normalize gait cycles to $100 \%$, was done with a piecewise cubic spline to interpolate the data at $1 \%$ intervals. The data were then averaged in $10 \%$ intervals, and the first and last interval were evaluated to determine the effects of repetitive drop jumps on impact phase joint mechanics.

\section{Statistical Analysis}

Dependent variables analyzed in the current study included DJH, peak VGRF, trunk and lower extremity joint positions at initial contact, range of motion (ROM) calculated from touchdown to maximum flexion during the impact phase, and maxima and minima from the joint power profiles during the impact phase. Peak joint powers identified for analysis included the initial hip power generation peak (HP1) and absorption peak (HP2), the first and second knee power absorption peaks (KP1 and KP2, respectively) and the peak ankle power absorption (AP) (Decker et al., 2003; Zhang et al., 2000). These kinematic and kinetic variables were compared using paired $t$ tests. In addition, a $2 \times 3$ repeated-measures ANOVA (fatigue $\times$ joint) was performed for to analyze the difference between the absolute energy absorption at the of hip, knee, and ankle joints during the impact phase. In the event of a significant interaction paired $t$ tests were used to test 1) comparisons across fatigue separately for each joint and 2) comparisons across joint separately for unfatigued and fatigued. Significance for all tests was set at $p<.05$, and all statistical analyses were performed using SPSS (version 17.0, SPSS Science Inc, Chicago, IL, USA).

\section{Results}

Although twelve subjects were recruited and participated in this study, data for three subjects were later excluded due to failure to demonstrate a $20 \%$ decrement in $\mathrm{DJH}$
Table 1 Demographic information of the subjects who participated in the study

\begin{tabular}{lccccc}
\hline Subject & Gender & $\begin{array}{c}\text { Age } \\
(\mathbf{y r})\end{array}$ & $\begin{array}{c}\text { Height } \\
(\mathbf{m})\end{array}$ & $\begin{array}{c}\text { Weight } \\
(\mathbf{k g})\end{array}$ & \# DJ \\
\hline 1 & $\mathrm{~F}$ & 22 & 1.73 & 72.0 & 118 \\
$2^{*}$ & $\mathrm{~F}$ & 21 & 1.65 & 62.6 & 200 \\
$3 *$ & $\mathrm{~F}$ & 23 & 1.63 & 52.3 & 200 \\
4 & $\mathrm{~F}$ & 21 & 1.63 & 52.1 & 130 \\
5 & $\mathrm{~F}$ & 23 & 1.73 & 65.7 & 164 \\
6 & $\mathrm{~F}$ & 23 & 1.57 & 54.3 & 163 \\
7 & $\mathrm{M}$ & 22 & 1.96 & 127.5 & 133 \\
$8 *$ & $\mathrm{M}$ & 24 & 1.60 & 67.8 & 200 \\
9 & $\mathrm{M}$ & 21 & 1.93 & 81.1 & 160 \\
10 & $\mathrm{M}$ & 23 & 1.83 & 87.8 & 134 \\
11 & $\mathrm{M}$ & 19 & 1.83 & 81.8 & 155 \\
12 & $\mathrm{M}$ & 25 & 1.73 & 73.5 & 143 \\
Mean & & 22.3 & 1.74 & 73.2 & 158 \\
$S D$ & & 1.6 & 0.13 & 20.8 & 29 \\
\hline
\end{tabular}

Note. *Excluded from data analysis.

(Table 1). The remaining subjects performed an average of $148.9 \pm 24.7$ drop jumps from an average height of $37.8 \pm 5.7 \mathrm{~cm}$ during the experimental protocol and exhibited a significant decrease in jump height. Based on these results, the drop jumps performed during the initial $10 \%$ of the experimental protocol will be characterized as unfatigued, and drop jumps during the final $10 \%$ will be characterized as fatigued. Ensemble averages to demonstrate the general effect of fatigue for VGRF, joint kinematics and joint powers are graphically represented in Figures 1-3 respectively.

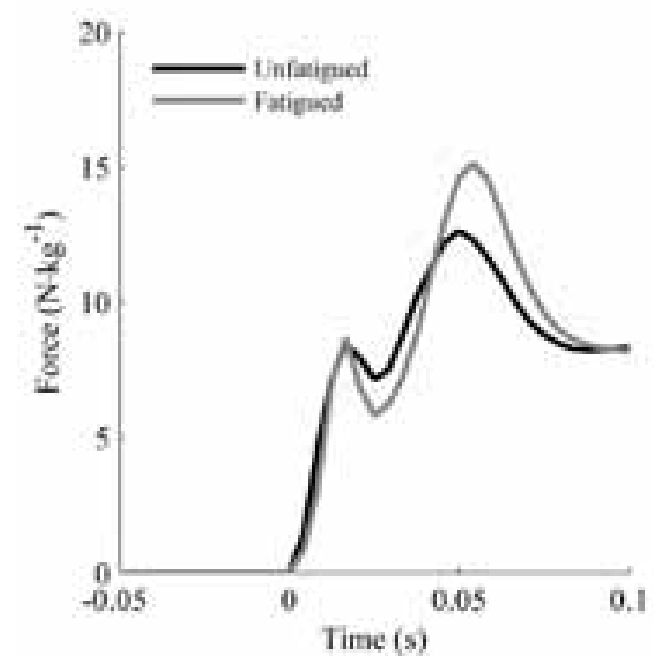

Figure 1 - Group mean VGRF curves during the impact phase from unfatigued (solid black line), and fatigued (gray line). Zero time represents initial ground contact. 

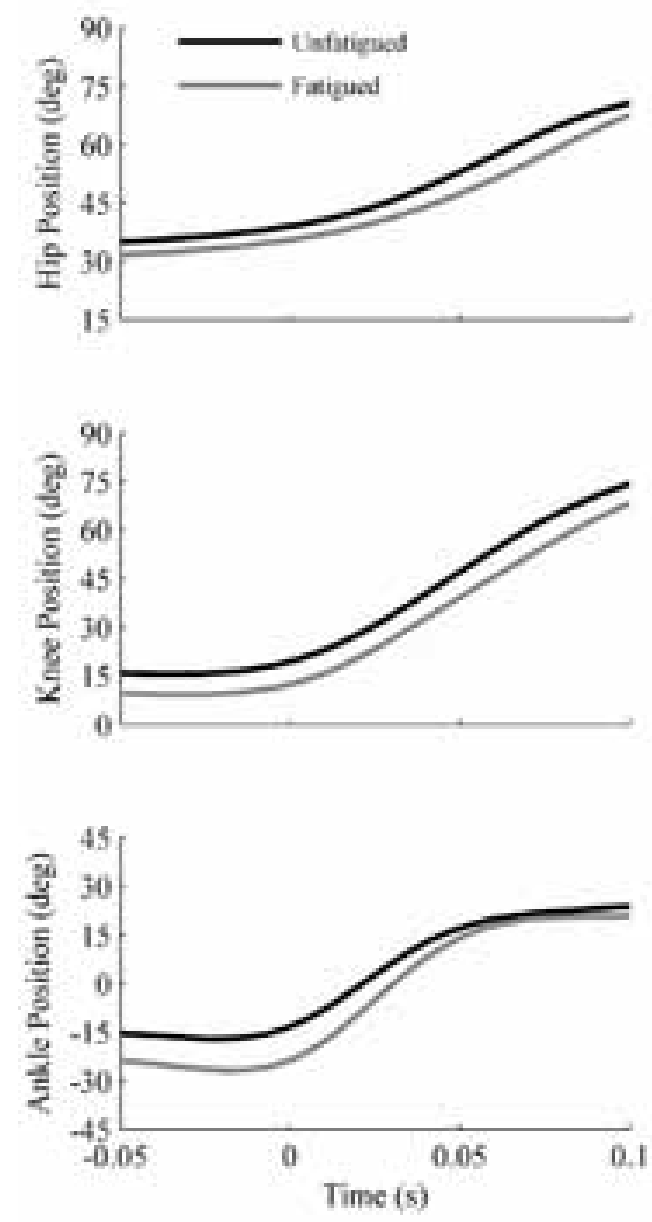

Figure 2 - Group mean hip, knee, and ankle position curves during the impact phase from unfatigued (solid black line) and fatigued (gray line), Positive values indicate trunk, hip and knee flexion, and ankle dorsiflexion joint rotations. Zero time represents initial ground contact.

There was no significant difference in peak VGRF as a result of fatigue (Table 2). However, as a result of fatigue, individuals landed with significant increases of $7.0^{\circ}$ knee flexion and $10.6^{\circ}$ ankle plantar flexion at initial ground contact. In addition, subjects performed drop jumps with significant increases of $2.6^{\circ}$ trunk flexion ROM and $7.5^{\circ}$ ankle plantar flexion ROM as a result of fatigue. Joint power analysis revealed that fatigue resulted in a significant increase of $2.1 \mathrm{~W} / \mathrm{kg}$ in peak power absorption at the ankle.

The fatigue $\times$ joint interaction for impact phase energy absorption was significant (Table 3 ). Post hoc analyses revealed that during unfatigued drop jumps individuals absorbed more energy at the knee when compared with both the hip $[p<.001]$ and ankle $[p<.001]$, and that this relationship held constant during fatigued drop jumps. However, as a result of fatigue there was an increase in absolute energy absorption at the ankle $[p=$ $.003]$ which was accompanied by an approximately equal reduction in energy absorption at the knee [ $p=.008]$.
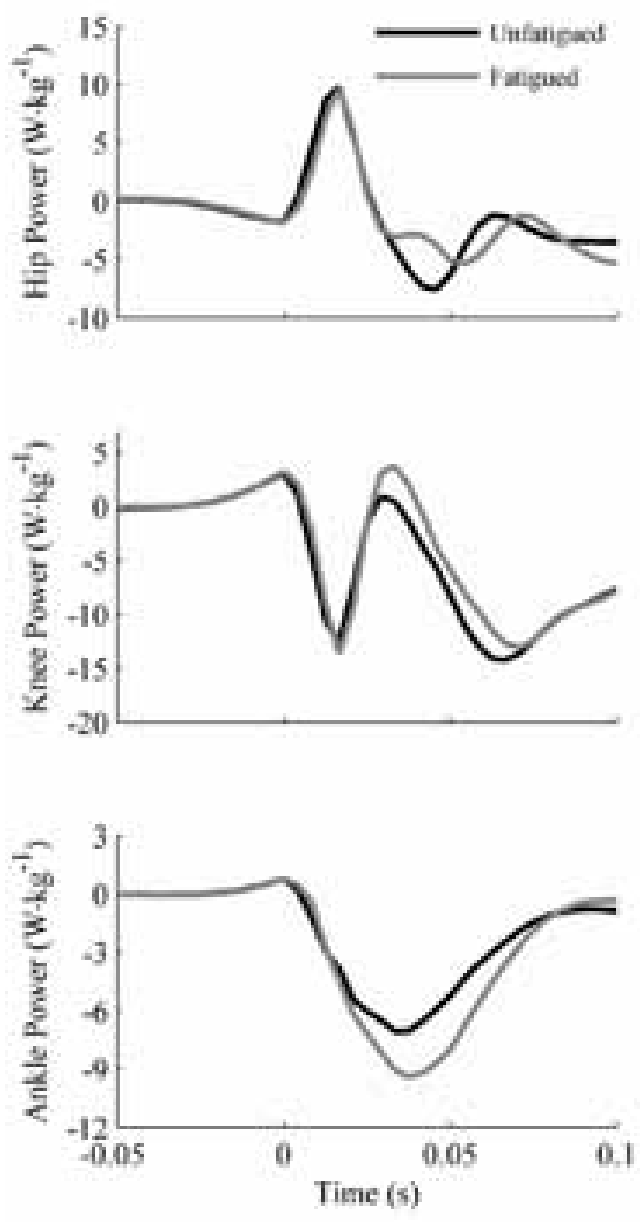

Figure 3 - Group mean hip, knee, and ankle power curves during the impact phase from unfatigued (solid black line) and fatigued (gray line), Positive values correspond to power generation. Zero time represents initial ground contact.

\section{Discussion}

The objective of this study was to investigate the effects of fatigue on lower extremity joint kinematics, and kinetics during repetitive drop jumps. During landing, the lower extremity joints function to reduce and control the downward momentum acquired during the flight phase. It has been suggested that lower peak VGRF indicates a safer landing strategy, while high peak VGRF can lead to injuries (Dufek \& Bates, 1990; Pappas et al., 2007). Our data suggest that fatigue does not result in an increase in the peak VGRF during drop jumping. These findings were not expected considering that fatigue resulted in more extended joint positions at initial contact. Previous research has reported greater peak VGRF during stiff landings, which are characterized by increased joint extension (Bobbert et al., 1987a; Bobbert et al., 1987b; Bobbert et al., 1986; DeVita \& Skelly, 1992; Zhang et al., 2000). The increased ankle plantar flexion as a result of fatigue observed may explain this difference as 
Table 2 Means $\pm S D$ for fatigue effects, as well as $p$-values and effect sizes (ES)

\begin{tabular}{|c|c|c|c|c|c|}
\hline & Unfatigued & Fatigued & $p$ & ES & $95 \% \mathrm{Cl}$ \\
\hline DJH $(\mathrm{cm}) *$ & $26.0 \pm 5.3$ & $21.7 \pm 4.1$ & $<0.001$ & 0.92 & 0.01 \\
\hline pVGRF (N/kg) & $20.2 \pm 5.3$ & $19.4 \pm 5.4$ & 0.570 & 0.13 & 1.44 \\
\hline \multicolumn{6}{|c|}{ Initial Joint Position (degrees) } \\
\hline Trunk & $18.5 \pm 5.4$ & $19.5 \pm 7.7$ & 0.503 & 0.17 & 1.26 \\
\hline Hip & $39.0 \pm 7.1$ & $35.4 \pm 7.7$ & 0.095 & 0.46 & 1.65 \\
\hline Knee * & $19.3 \pm 7.1$ & $12.3 \pm 6.0$ & 0.022 & 1.06 & 1.90 \\
\hline Ankle * & $-13.2 \pm 9.4$ & $-23.8 \pm 7.2$ & 0.009 & 1.27 & 2.35 \\
\hline \multicolumn{6}{|c|}{ Range of Motion (degrees) } \\
\hline Trunk * & $8.1 \pm 2.3$ & $10.7 \pm 2.9$ & 0.007 & 0.99 & 0.69 \\
\hline Hip & $31.7 \pm 6.8$ & $32.1 \pm 6.0$ & 0.639 & 0.01 & 0.91 \\
\hline Knee & $54.9 \pm 4.9$ & $55.9 \pm 5.1$ & 0.267 & 0.20 & 0.91 \\
\hline Ankle * & $37.1 \pm 9.3$ & $44.6 \pm 6.8$ & 0.024 & 0.92 & 2.13 \\
\hline \multicolumn{6}{|c|}{ Peak Joint Powers (W/kg) } \\
\hline HP1 & $13.6 \pm 4.8$ & $11.6 \pm 4.9$ & 0.189 & 0.41 & 1.48 \\
\hline HP2 & $-18.1 \pm 7.2$ & $-16.0 \pm 6.2$ & 0.283 & 0.31 & 1.92 \\
\hline KP1 & $-15.9 \pm 5.3$ & $-16.4 \pm 5.9$ & 0.687 & 0.09 & 1.13 \\
\hline KP2 & $-17.3 \pm 4.7$ & $-16.5 \pm 3.2$ & 0.441 & 0.20 & 1.14 \\
\hline $\mathrm{AP} *$ & $-8.1 \pm 3.4$ & $-10.2 \pm 2.6$ & 0.007 & 0.69 & 0.76 \\
\hline
\end{tabular}

Note. *Indicates significant fatigue main effect $(p<0.05)$.

Table 3 Absolute impact phase joint work (J/kg)

\begin{tabular}{lll|lccc}
\hline & Unfatigued $^{\mathrm{a}, \mathrm{b}}$ & Fatigued $^{\mathrm{a}, \mathrm{b}}$ & & $\boldsymbol{F}$ & $\boldsymbol{p}$ & ES \\
\hline Hip & $-0.37 \pm 0.11$ & $-0.36 \pm 0.09$ & Fatigue $\times$ Joint & 15.193 & $<0.001$ & 0.655 \\
Knee $\dagger$ & $-0.81 \pm 0.18$ & $-0.73 \pm 0.14$ & Fatigue & 0.005 & 0.945 & 0.001 \\
Ankle $\dagger$ & $-0.33 \pm 0.15$ & $-0.43 \pm 0.13$ & Joint & 32.799 & $<0.001$ & 0.804 \\
\hline
\end{tabular}

${ }^{a}$ Indicates that the knee is significantly different from the hip.

${ }^{\mathrm{b}}$ Indicates that the knee is significantly different from the ankle.

$\dagger$ Indicates that unfatigued is significantly different from fatigued.

increased plantar flexion at ground contact results in the greatest amount of shock absorption and reduced peak VGRF (Self \& Paine, 2001). Therefore, the increased knee extension when fatigued is compensated for by an increased use of the ankle plantar flexors to help absorb the forces during impact, resulting in similar peak VGRF.

The drop jump task individuals were asked to perform required an immediate vertical jump after landing, with the intent of achieving maximum height as quickly as possible. As individuals fatigued, the knee extensors were unable to effectively handle the landing impact and the contribution of the ankle plantar flexors to absorbing the impact increased. While the increased contribution of the ankle plantar flexors act as a supplementary mechanism for impact absorption it potentially limits performance.
It has been documented that both the stretch reflex and associated muscle stiffness contribute to performance in stretch shorten cycle exercises such as a drop jump (Komi \& Gollhofer, 1997). Fatigue induced via repetitive stretch shorten cycles similar to the drop jump task used in the current study, has been shown to decrease the stretch-reflex sensitivity (Horita et al., 1996; Nicol et al., 1996). Assuming that the muscle damage was indeed severe enough in the current study, it may have disturbed muscle stiffness and the stretch-reflex mechanism, which are critical in stretch-shorten cycle performance (Horita et al., 2003). It has also been suggested that drop jump performance is determined by prelanding muscle activity (Dietz et al., 1981; Horita et al., 2003; Horita et al., 2002). This preactivation is believed to be involved in the centrally preprogrammed motor commands of the 
required motor tasks (Dietz et al., 1981; Dyhre-Poulsen et al., 1991; Gollhofer \& Kyrolainen, 1991) as well as compensation for local muscular failure after fatigue (Horita et al., 2003; Horita et al., 1996; Horita et al., 2002).

In addition to the interaction between prelanding muscle activity and muscle stiffness, two distinct jumping strategies influence performance during drop jumps: 1) the "bouncing" technique, and 2) the "absorbing" technique (Bobbert et al., 1987a; Bobbert et al., 1986; Horita et al., 2002). The "bouncing" technique is associated with high initial knee joint stiffness (Horita et al., 2002) where stretch reflex potentiation could assist in the production of a higher takeoff velocity (Komi \& Gollhofer, 1997). On the other hand, the "absorbing" technique is characterized by a rapid decrease of initial VGRF peak followed by lower VGRF levels at the end of the stretch (Horita et al., 2002). A high initial peak followed by a sharp drop in VGRF has been reported after repeated stretch-shorten cycle action in drop jumps (Horita et al., 2002), arm exercises (Gollhofer et al., 1987) and marathon running (Avela \& Komi, 1998; Nicol et al., 1991). As individuals fatigue and joint stiffness changes, the initial peak VGRF at ground contact becomes intolerable and therefore must be absorbed, reducing performance (Gollhofer et al., 1987; Horita et al., 2002; Nicol et al., 1991).

Fatigue not only impairs performance, but also impairs proprioceptive acuity and reduces preactivation of stabilizing muscles (Lephart \& Fu, 1995). Thus, landing mechanics in the presence of fatigue may result in a loss of balance, which could stress and injure soft tissues, particularly ligamentous structures (Lephart \& Fu, 1995). The knee joint is particularly susceptible to traumatic injury because it is located at the ends of two long lever arms, the tibia and the femur. In addition, knee joint stability is determined by an interaction of passive restraints produced by the ligaments and other joint structures, joint geometry, friction between cartilage surfaces and stability provided by muscles acting on the joint (Johansson et al., 2000). Of all these factors the stability provided by the contracting muscles appears to be the most important for knee stability (Hewett et al., 2000). Eccentric muscle action stabilizes the knee joint dynamically as eccentric muscle contractions act to control deceleration of body segments during dynamic tasks (Johansson et al., 2000). Therefore, as evident by the results of the current study, the muscular work needed to control the knee joint is greater than that needed to control the hip or ankle joints and potentially increases injury risk (Decker et al., 2003; Lephart \& Fu, 1995).

The increased knee extension and ankle plantar flexion observed at initial contact may have relevance in terms of injury risk as increased plantar flexion may be a compensatory mechanism for the reduced capability of the knee extensors to decelerate the body and absorb the landing impact. However, landing with the knee close to full extension has been suggested to be a predisposing factor to knee injury (Decker et al., 2003; Huston et al., 2001). Pandy and Shelburne (1997) concluded that the ACL may experience larger forces near full extension of the knee because the hamstrings are less capable of counterbalancing anterior translation of the tibia caused by the quadriceps. In addition, Markolf et al. (2004) demonstrated that ACL forces caused by quadriceps contraction increase in the last $40^{\circ}$ of knee extension, peaking close to full extension.

In the current study, the knee extensors were the muscle group primarily responsible for reducing the body's kinetic energy. However, as individuals became fatigued, the energy absorption at the knee decreased with an approximately equal increase in energy absorption at the ankle. This result suggests that individuals adopted a landing strategy that shifted a greater burden to the ankle for absorbing the kinetic energy of the impact as they fatigued. These results are consistent with previous research (DeVita \& Skelly, 1992; Zhang et al., 2000) that reported a greater relative contribution of the ankle plantar flexors as knee extension angles increased at initial ground contact.

Peak joint power values can also be used to describe the mechanisms by which kinetic energy is dissipated during landing (DeVita \& Skelly, 1992). Although neither of the peak hip powers (HP1 or HP2) were statistically significant, the decrease in initial peak power generation at the hip (HP1) was accompanied by an equal reduction in peak power absorption at the hip (HP2). DeVita and Skelly (1992) suggested that the functional role of HP1 and HP2 may be to control the acceleration of the trunk so that so that the mass of the upper body is closer horizontally to the knee joint, effectively reducing the external flexion moment at the knee and the subsequent load on the knee extensors. There is evidence that active trunk flexion during landing alters lower extremity kinematics and energy absorption in a manner which potentially reduces ACL loading and injury risk (Blackburn \& Padua, 2008; Kulas et al., 2008; Kulas et al., 2010). Kulas et al. (2010) evaluated the effects of added trunk load and adaptations to trunk position on knee anterior shear force and muscle forces during landing. They reported that individuals who responded to added trunk load with active trunk flexion performed landings with increased hamstrings muscle force and decreased knee anterior shear forces. In the current study, individuals exhibited a $2.6^{\circ}$ increase in trunk flexion ROM when fatigued (unfatigued $=8.1^{\circ}$; fatigued $=10.7^{\circ}$ ). Thus, the observed decreases in HP1 and HP2 as individuals fatigued may indicate an active control of the trunk to reduce the load on the knee extensors and prevent injury to the soft tissues, particularly ligamentous structures. Further investigation is warranted to understand the influence of the upper body on lower extremity neuromechanics and possible links with injury risk.

While our results indicate that landing mechanics were altered as a result of fatigue there are several methodological limitations to our study that could have contributed to this outcome. A first limitation of the study was that it was performed in a controlled laboratory environment where subjects knew exactly what to expect. Although this allows accurate comparisons between conditions, it does not closely simulate athletic 
competition. Most noncontact injuries involve unexpected factors during a game or practice such as motion of an opponent, or miscalculation of ball motion that would make landings more unpredictable than laboratory settings. Furthermore, the drop jump task itself was not identical to movements observed during athletic participation since we constrained the use of the arms during the drop jumps. All subjects were recreationally active, participating in competitive intramural and/or recreational sports sponsored by the university at least three times per week. However, this does not insure equal ability in performing drop jumps and some subjects might have been more skillful than others. A final limitation of the current study was that three subjects were excluded from data analysis. These subjects were excluded because it was determined a priori that completion of 200 drop jumps without a $20 \%$ decrement in DJH suggested less than full effort throughout the protocol. While it is possible that these subjects were extremely fatigue resistant, their data were not representative of those who exhibited a fatigue induced change in performance, which was the focus of the current study.

In conclusion, while the knee extensors were the muscle group primarily responsible for absorbing the impact, individuals compensated for increased knee extension when fatigued by an increased use of the ankle plantar flexors to help absorb the forces during impact. Our observation of increased ankle ROM and energy absorption at the ankle, coupled with decreased energy absorption at the knee, suggests an altered landing strategy as individuals became fatigued. While increased knee extension has been suggested to be a predisposing factor to knee injury, a landing strategy incorporating large ankle ROM affords greater shock absorption at the ankle joint (Self \& Paine, 2001) and hence, minimizes energy transfer to the knee joint (Decker et al., 2003). Whether this strategy alters injury risk is difficult to elucidate from the current data, and future research is necessary to determine influence of increased ankle plantar flexor contribution on lower extremity injury risk.

\section{References}

Avela, J., \& Komi, P.V. (1998). Reduced stretch reflex sensitivity and muscle stiffness after long-lasting stretchshortening cycle exercise in humans. European Journal of Applied Physiology and Occupational Physiology, 78(5), 403-410.

Blackburn, J.T., \& Padua, D.A. (2008). Influence of trunk flexion on hip and knee joint kinematics during a controlled drop landing. Clinical Biomechanics (Bristol, Avon), 23(3), 313-319.

Bobbert, M.F., Huijing, P.A., \& van Ingen Schenau, G.J. (1987a). Drop jumping. I. The influence of jumping technique on the biomechanics of jumping. Medicine and Science in Sports and Exercise, 19(4), 332-338.

Bobbert, M.F., Huijing, P.A., \& van Ingen Schenau, G.J. (1987b). Drop jumping. II. The influence of dropping height on the biomechanics of drop jumping. Medicine and Science in Sports and Exercise, 19(4), 339-346.
Bobbert, M.F., Mackay, M., Schinkelshoek, D., Huijing, P.A., \& van Ingen Schenau, G.J. (1986). Biomechanical analysis of drop and countermovement jumps. European Journal of Applied Physiology and Occupational Physiology, 54(6), 566-573.

Chappell, J.D., Herman, D.C., Knight, B.S., Kirkendall, D.T., Garrett, W.E., \& Yu, B. (2005). Effect of fatigue on knee kinetics and kinematics in stop-jump tasks. American Journal of Sports Medicine, 33(7), 1022-1029.

Coventry, E., O'Connor, K.M., Hart, B.A., Earl, J.E., \& Ebersole, K.T. (2006). The effect of lower extremity fatigue on shock attenuation during single-leg landing. Clinical Biomechanics (Bristol, Avon), 21(10), 1090-1097.

Davis, R.B., Ounpuu, S., Tyburski, D., \& Gage, J.R. (1991). A gait analysis data collection and reduction technique. Human Movement Science, 10(5), 575-587.

Decker, M.J., Torry, M.R., Wyland, D.J., Sterett, W.I., \& Richard Steadman, J. (2003). Gender differences in lower extremity kinematics, kinetics and energy absorption during landing. Clinical Biomechanics (Bristol, Avon), 18(7), 662-669.

Dempster, W.T. (1955). Space requirements of the seated operator. Ohio: Wright-Patterson Airforce Base.

DeVita, P., \& Skelly, W.A. (1992). Effect of landing stiffness on joint kinetics and energetics in the lower extremity. Medicine and Science in Sports and Exercise, 24(1), 108-115.

Dietz, V., Noth, J., \& Schmidtbleicher, D. (1981). Interaction between pre-activity and stretch reflex in human triceps brachii during landing from forward falls. The Journal of Physiology, 311, 113-125.

Dufek, J.S., \& Bates, B.T. (1990). The evaluation and prediction of impact forces during landings. Medicine and Science in Sports and Exercise, 22(3), 370-377.

Dyhre-Poulsen, P., Simonsen, E.B., \& Voigt, M. (1991). Dynamic control of muscle stiffness and $\mathrm{H}$ reflex modulation during hopping and jumping in man. The Journal of Physiology, 437, 287-304.

Fagenbaum, R., \& Darling, W.G. (2003). Jump landing strategies in male and female college athletes and the implications of such strategies for anterior cruciate ligament injury. American Journal of Sports Medicine, 31(2), 233-240.

Gollhofer, A., Komi, P.V., Miyashita, M., \& Aura, O. (1987). Fatigue during stretch-shortening cycle exercises: Changes in mechanical performance of human skeletal muscle. International Journal of Sports Medicine, 8(2), 71-78.

Gollhofer, A., \& Kyrolainen, H. (1991). Neuromuscular control of the human leg extensor muscles in jump exercises under various stretch-load conditions. International Journal of Sports Medicine, 12(1), 34-40.

Hawkins, R.D., Hulse, M.A., Wilkinson, C., Hodson, A., \& Gibson, M. (2001). The association football medical research programme: an audit of injuries in professional football. British Journal of Sports Medicine, 35(1), 43-47.

Hewett, T.E., Paterno, M.V., \& Noyes, F.R. (2000). Neuromuscular contributions to knee kinematics and kinetics: Normal versus pathological state. Proprioception and Neuromuscular Control in Joint Stability. S. M. Lephart and F. H. Fu. Champaign: Human Kinetics.

Horita, T., Komi, P.V., Hamalainen, I., \& Avela, J. (2003). Exhausting stretch-shortening cycle (SSC) exercise causes greater impairment in SSC performance than in pure concentric performance. European Journal of Applied Physiology, 88(6), 527-534.

Horita, T., Komi, P.V., Nicol, C., \& Kyrolainen, H. (1996). Stretch shortening cycle fatigue: interactions among joint 
stiffness, reflex, and muscle mechanical performance in the drop jump [corrected]. European Journal of Applied Physiology and Occupational Physiology, 73(5), 393-403.

Horita, T., Komi, P.V., Nicol, C., \& Kyrolainen, H. (2002). Interaction between pre-landing activities and stiffness regulation of the knee joint musculoskeletal system in the drop jump: implications to performance. European Journal of Applied Physiology, 88(1-2), 76-84.

Huston, L.J., Vibert, B., Ashton-Miller, J.A., \& Wojtys, E.M. (2001). Gender differences in knee angle when landing from a drop-jump. The American Journal of Knee Surgery, 14(4), 215-219, discussion 219-220.

Johansson, H., Pedersen, J., Bergenheim, M., \& Djupsjobacka, M. (2000). Peripheral afferents of the knee: Their effects on central mechanisms regulating muscle stiffness, joint stability, and proprioception and coordination. Proprioception and Neuromuscular Control in Joint Stability. S. M. Lephart and F. H. Fu. Champaign: Human Kinetics.

Kadaba, M.P., Ramakrishnan, H.K., \& Wootten, M.E. (1990). Measurement of lower extremity kinematics during level walking. Journal of Orthopaedic Research, 8(3), 383-392.

Kernozek, T.W., Torry, M.R., \& Iwasaki, M. (2008). Gender differences in lower extremity landing mechanics caused by neuromuscular fatigue. American Journal of Sports Medicine, 36(3), 554-565.

Komi, P.V., \& Gollhofer, A. (1997). Stretch reflexes can have an important role in force enhancement during SSC exercise. Journal of Applied Biomechanics, 13(4), 451-460.

Kulas, A., Zalewski, P., Hortobagyi, T., \& DeVita, P. (2008). Effects of added trunk load and corresponding trunk position adaptations on lower extremity biomechanics during drop-landings. Journal of Biomechanics, 41(1), 180-185.

Kulas, A.S., Hortobagyi, T., \& Devita, P. (2010). The interaction of trunk-load and trunk-position adaptations on knee anterior shear and hamstrings muscle forces during landing. Journal of Athletic Training, 45(1), 5-15.

Kulas, A.S., Schmitz, R.J., Schultz, S.J., Watson, M.A., \& Perrin, D.H. (2006). Energy absorption as a predictor of leg impedance in highly trained females. Journal of Applied Biomechanics, 22(3), 177-185.

Lephart, S.M., \& Fu, F.H. (1995). The role of proprioception in the treatment of sports injuries. Sports Exercise Injury, $1,96-102$.

Madigan, M.L., \& Pidcoe, P.E. (2003). Changes in landing biomechanics during a fatiguing landing activity. Journal of Electromyography and Kinesiology, 13(5), 491-498.

Markolf, K.L., O’Neill, G., Jackson, S.R., \& McAllister, D.R. (2004). Effects of applied quadriceps and hamstrings muscle loads on forces in the anterior and posterior cru- ciate ligaments. American Journal of Sports Medicine, 32(5), 1144-1149.

McLean, S.G., Fellin, R.E., Suedekum, N., Calabrese, G., Passerallo, A., \& Joy, S. (2007). Impact of fatigue on gender-based high-risk landing strategies. Medicine and Science in Sports and Exercise, 39(3), 502-514.

Moran, K.A., \& Marshall, B.M. (2006). Effect of fatigue on tibial impact accelerations and knee kinematics in drop jumps. Medicine and Science in Sports and Exercise, 38(10), 1836-1842.

Nicol, C., Komi, P.V., Horita, T., Kyrolainen, H., \& Takala, T.E (1996). Reduced stretch-reflex sensitivity after exhausting stretch-shortening cycle exercise. European Journal of Applied Physiology and Occupational Physiology, 72(56), 401-409.

Nicol, C., Komi, P.V., \& Marconnet, P. (1991). Fatigue effects of marathon running on neuromuscular performance I. changes in muscle force and stiffness characteristics. Scandinavian Journal of Medicine \& Science in Sports, 1, 10-17.

Pandy, M.G., \& Shelburne, K.B. (1997). Dependence of cruciate-ligament loading on muscle forces and external load. Journal of Biomechanics, 30(10), 1015-1024.

Pappas, E., Sheikhzadeh, A., Hagins, M., \& Nordin, M. (2007). The effect of gender and fatigue on the biomechanics of bilateral landings from a jump: Peak values. Journal of Sports, Science, and Medicine, 6(1), 77-84.

Rahnama, N., Reilly, T., \& Lees, A. (2002). Injury risk associated with playing actions during competitive soccer. British Journal of Sports Medicine, 36(5), 354-359.

Schot, P.K., Bates, B.T., \& Dufek, J.S. (1994). Bilateral performance symmetry during drop landing: a kinetic analysis. Medicine and Science in Sports and Exercise, 26(9), 1153-1159.

Schot, P.K., \& Dufek, J.S. (1993). Landing performance, Part I: Kinematic, kinetic and neuromuscular aspects. Medicine, Exercise. Nutrition and Health (Berkhamsted, Hertfordshire), 2, 69-83.

Self, B.P., \& Paine, D. (2001). Ankle biomechanics during four landing techniques. Medicine and Science in Sports and Exercise, 33(8), 1338-1344.

Woltring, H.J. (1986). A Fortran package for generalized, crossvalidatory spline smoothing and differentiation. Advances in Engineering Software, 8(2), 104-107.

Zhang, S.N., Bates, B.T., \& Dufek, J.S. (2000). Contributions of lower extremity joints to energy dissipation during landings. Medicine and Science in Sports and Exercise, 32(4), 812-819. 